MOLPHARM/2004/010348

\title{
An orphan receptor adopted by a family of transmitters
}

Olivier Civelli

Department of Pharmacology and Developmental and Cell Biology, University of California, Irvine, Irvine, California

Address: Department of Pharmacology, Med Surge II, Room 369 Irvine, CA 92697-4625

Email ocivelli@uci.edu 
Molecular Pharmacology Fast Forward. Published on December 16, 2004 as DOI: 10.1124/mol.104.010348

This article has not been copyedited and formatted. The final version may differ from this version.

MOLPHARM/2004/010348

Abstract:

A tenet of pharmacology states that a receptor must be selective for and bind its natural ligand with high affinity. The recently-discovered ligands of some deorphanized GPCRs challenge this concept. 
MOLPHARM/2004/010348

Pharmacological research aims at investigating the natural specificity of intermolecular recognition processes. We find a high degree of selectivity between transmitters (primary messengers) and their receptors. For example, dopamine receptors are entities different from adrenergic receptors, although their transmitters are chemically very similar. On the other hand, the fact that a small molecule is a transmitter does not imply that a structurally related one will serve the same function, e.g., glutamate is a transmitter while aspartate is not (thus far). Nature has gone a long way in establishing selectivity in receptor recognition. Now, several recent discoveries challenge that concept.

In this issue of Molecular Pharmacology, Wellendorph et al (2004) report that a G protein-coupled receptor (GPCR) is not selective when it comes to natural ligand recognition. It can be activated by a series of basic $L$ - $\alpha$-amino acids with only a preference for basic amino acids.

To reach this conclusion the authors investigated a family C GPCR, called GPRC6A. The hallmark of the GPCRs from the family $\mathrm{C}$ is their long extracytoplasmic N-termini that bind small transmitters. The family includes, in human, eight metabotropic glutamate (mGluRs), two GABA-B, three taste, one calcium-sensing and six orphan receptors (Bockaert and Pin, 1999). GPRC6A was one of the orphan GPCRs; its highest degree of sequence similarity was not to a human receptor but instead, to one from goldfish, called 5.24 (Speca et al, 1999), which was known to sense basic amino acids, potent odorants in fish. Expecting that the amino terminal domain would be of primary importance in recognizing the GPRC6A transmitter, the authors generated a three-dimensional model of that domain using X-ray crystal data of the mGluR1 N-terminus as template. They 
MOLPHARM/2004/010348

predicted that the GPRC6A N-terminal domain would not recognize glutamate, but could accomodate binding of $\alpha$-amino acids with aliphatic side chains possessing strong distal hydrogen bond donors instead of amino acids with hydrophobic or acidic side chains.

To test their hypothesis, the authors set out to identify the GPRC6A transmitter by pharmacological means. They immediately encountered an issue that is becoming frequent in the orphan GPCR field: the lack of membrane expression for some exogenously transfected GPCRs. The authors struggled with this issue until they found that an engineered chimeric receptor (h6A/5.24), comprised of the $\mathrm{N}$-terminus of GPRC6A and the transmembrane and C-terminal domain of the goldfish 5.24 receptor, was able to be expressed at the cell surface. Knowing that 5.24 couples to $\mathrm{Gq}$ and that it would be impractical to exclude essential amino acids from culture medium if they were to employ mammalian cells, the authors used the Xenopus oocyte system, as an assay for receptor activity. They expressed h6A/5.24 and found that it could be activated at 100 $\mu \mathrm{M}$ not only by basic but also several neutral aliphatic L-a-amino acids. When they lowered the concentration to $10 \mu \mathrm{M}$ only L-Arg, L-Lys and L-Orn showed activation. At 3 $\mu \mathrm{M}$ none were active. The authors also cloned the mouse GPRC6A which, in contrast to its human counterpart, reaches the cell membrane upon exogenous transfection and found that it exhibits a similar pharmacological profile. But, while they were getting a hint at the pharmacological profile of the GPRC6A receptor the authors also faced the fact that oocytes are not an ideal assay for carrying out quantitative pharmacology (Pin et al 1992).

Testing amino acid receptors expressed in mammalian cells was problematicl because of the presence of amino acids in the medium. As a way to circumvent this problem, the 
MOLPHARM/2004/010348

authors used a procedure previously developed by Kuang et al (2003), which consists in preincubating mammalian cells in a calcium buffer devoid of amino acids. This protocol presumably resensitizes the receptor and allows for quantitative measurements of its response, in this case so the authors could determine that the h6A/5.24 receptor is activated by L-a-amino acids with a rank order of potency of L-Arg $(44 \mu \mathrm{M})>\mathrm{L}-\mathrm{Orn}$. LLys, L-Ala, L-Gly, L-Cit $(100-300 \mu \mathrm{M})>$ L-Gln, L-Ser, L-Met $(500-1000 \mu \mathrm{M})$. The remaining $L-\alpha$-amino acids were practically inactive, as were $D$-amino acids, some other transmitters such as GABA, serotonin and vasopressin and some metabolic intermediates such as creatine, spermine and urea. Thus, GPRC6A is undoubtedly a promiscuous receptor when it comes to ligand specificity.

These results lead to the question of the physiological role of GPRC6A. Because its sequence is most closely similar to the 5.24 fish receptor it could act as an olfactory receptor. But this is refuted on the basis of a Northern blot analysis (Wellendorph and Brauner-Osborne, 2004) that shows GPRC6A is expressed in the brain and in a variety of peripheral organs, including skeletal muscle, testis, leukocytes, heart and kidney. The authors therefore favor another hypothesis, that GPRC6A may function as a regulatory component of the urea cycle, since its primary ligands belong to that pathway. There is a precedent for such a claim: succinate and a-ketoglutarate have been paired to particular GPCRs (He et al, 2004). This hypothesis however faces a challenge since it implies that urea or citric cycle intermediates have to act as primary messengers. This raises two possibilities: either the intermediates act intracellularly, but then one asks how can the GPCRs induce second messengers, or the intermediates are secreted, but then, how is their release regulated?. 


\section{MOLPHARM/2004/010348}

The first possibility seems improbable since the GPCRs would have to embark on a thus far undescribed secretory pathway that inserts them into the mitochondrial inner or outer membranes. Furthermore, this would have to leave the $G$ proteins and the rest of the signaling machinery in the space between these membranes. The second possibility too butts against one issue: a molecule that participates in intermediate metabolism has to also act as a "transmitter," i.e. it has to be secreted in a regulatory manner. One hypothesis that might resolve this issue is to accept that the GPRC6A ligands are released directly from bursting cells and thus are used as monitors of cell death (Civelli 2005). But even this possibility is not without issues.

GPRC6A is activated by amino acids, in particular L-Arg, at about $50 \mu \mathrm{M}$. The concentration of L-Arg in the plasma is in the $100 \mu \mathrm{M}$ range. So, if Arg acts in an endocrine fashion, the receptor would be constantly and strongly activated. In addition, it would be surprising that any metabolic change would result in an high enough increase in the concentration of L-Arg to account for a significant difference in signaling. This suggests that L-Arg and the other GPRC6A ligands act in a paracrine fashion. The authors mention that GPRC6A, which belong to the family that include the calcium sensing receptor (Brown, 1993), may sense free amino acid concentrations. This discussion would support this hypothesis with the added possibility that it senses death of adjacent cells. Such a notion would also help explain the promiscuity of GPRC6A in ligand recognition. Thus, GPRC6A would have evolved to bind amino acids from the urea cycle (but not glutamate so as not to overlap with its neurotransmitter function). Demonstration of this hypothesis could prove difficult, since establishing GPRC6A knock-out mice might interfere with their development and administering exogenously one of the L-amino acid may require concentrations that will not reflect the paracrine 


\section{MOLPHARM/2004/010348}

nature of the expected response. The authors suggest a search for potent antagonists, and indeed, that may well be the best way to define the activity of this new receptor..

In summary, the manuscript by Wellendorph et al reports of the de-orphanization of the GPCR, GPRC6A. It is a successful effort in "reverse pharmacology" where ligands are paired to orphan GPCRs by random screening. In this case the choice of potential ligands was directed by structural analysis (and homology). But the discovery that GPRC6A is activated by $L-\alpha$ amino acids ultimately relied on screening. The results, however, leave open the possibility that other more potent ligand(s) may exist and that would oblige us to even further revise the concept of specificity in pharmacology. Further studies of GPCRC6A should thus prove of great interest. 
MOLPHARM/2004/010348

\section{$\underline{\text { References }}$}

Bockaert, J and Pin, J.P. (1999) Molecular tinkering of G protein-coupled receptors: an evolutionary success EMBO J 18:1723-1729

Brown, E.M. (1999) Physiology and pathophysiology of the extracellular calcium-sensing receptor Am.J.Med. 106:238-253

Civelli O. (2005) GPCR deorphanizations: the novel, the known and the unexpected transmitters Trends Pharm. Sci. (in press)

He, W., Miao, F.J.P., Lin, C.H., Schwandner, R.T., Wang, Z., Gao, J., Chen, J.H., Tian, $\mathrm{H}$. and Ling L. (2004) Citric acid cycle intermediates as ligands for orphan G-proteincoupled receptors Nature 429:188-193

Kuang D., Yao, Y., Wang, M., Pattabiraman, N., Kotra, L.P. and Hampson D.R. (2003) Molecular similarities in the ligand binding pockets of an odorant receptor and metabotropic glutamate receptors J. Biol. Chem 278:42551-42559

Pin, J.P., Waeber, C., Prezeau, L., Bockaert, J. and Heinemann S.F. (1992) Alternative splicing generates metabotropic glutamate receptors inducing different patterns of calcium release in Xenopus oocytes. Proc. Natl. Acad. Sci. USA 89:10331-10335

Speca,D.J., Lin, D.M., Sorensen P.W., Isacoff E.Y., Ngai, J. and Dittman A.H. Functional identification of a goldfish odorant receptor Neuron 23:487-498

Wellendorph, P., Hansen, K.B., Balsgaard, A., Greenwood, J.R., Egebjerg, J. and Brauner-Osborne, H. Deorphnaization of GPRC6A: a promiscuous L- $\alpha$-amino acid receptor with preference for basic amino acids. Mol. Pharm. this issue. 